\title{
Lévy Flights in Quantum Transport in Quasiballistic Wires
}

\author{
M. Leadbeater, ${ }^{1}$ V. I. Falko, ${ }^{2,3}$ and C. J. Lambert ${ }^{2}$ \\ ${ }^{1}$ Max-Planck-Institut für Physik komplexer Systeme, Nöthnitzer Strasse 38, 01187 Dresden, Germany \\ ${ }^{2}$ School of Physics and Chemistry, Lancaster University, Lancaster, LA1 4YB, United Kingdom \\ ${ }^{3}$ Département de Physique, Université Joseph Fourier, Grenoble 1, France
}

(Received 10 March 1998)

\begin{abstract}
Conductance fluctuations, localization, and statistics of Lyapunov exponents are studied numerically in pure metallic wires with rough boundaries (quasiballistic wires). We find that the correlation energy of conductance fluctuations scales anomalously with the sample dimensions, indicating the role of Lévy flights. Application of a magnetic field deflects the Lévy flights which reduces the localization length. This deflection also breaks geometrical flux cancellation and restores the usual Aharonov-Bohm type magnetoconductance fluctuations. [S0031-9007(98)06794-5]
\end{abstract}

PACS numbers: 72.10.Fk, 05.40. $+\mathrm{j}, 42.25 . \mathrm{Bs}, 73.23 .-\mathrm{b}$

During recent years theoretical interest in mesoscopic systems has started to divert from studies of universal properties of ergodic systems, such as disordered metals, towards structures which are nonergodic [1]. Ergodicity of diffusive conductors is provided by effective mixing of the phase space corresponding to the classical counterpart of quantum mechanics - due to quantum scattering at a short-range random potential. In nonergodic systems, homogeneous mixing of classical phase space does not occur before the quantum state is formed. Although issues of nonergodicity are usually addressed in a context of spectral and wave function statistics in closed systems, such as partly chaotic billiards [2-4], one may extend the notion of nonergodicity to open systems and study its implications in quantum transport effects such as localization and conductance fluctuations. We distinguish below a class of disordered objects where a large part of classical phase space is not mixed [5] since the particle motion through it is not diffusive. These systems are pure metallic wires with rough edges corrugated at the length scale of the electron wavelength (quasiballistic wires) [6-13], where the existence of anomalously long grazing ballistic paths is known to lead to noticeable effects even in classical transport properties [14].

In the theory of dynamically generated random walks, ballistic paths with lengths very much exceeding the average have the name Lévy flights $[15,16]$, and when the latter occur with a relatively high probability they determine a superdiffusion character of random motion. In diffusive wires with static short-range bulk impurities, the probability of finding a ballistic path $\eta$ longer than the mean free path $l$ is exponentially small, $\sim \exp (-\eta / l)$. This leads to the normal diffusion relation between the variance of the lengths of random walk paths and the system size and gives the "ergodic" form for the correlation energy of conductance fluctuations, $E_{\mathrm{c}} \sim h D / L^{2}[1]$. A natural example of a classically superdiffusive system with static disorder is a pure metallic wire with corrugated edges, where Lévy flights are just those grazing ballistic trajectories which cross it at a small angle, $\alpha \ll 1$, to the wire axis. In wires with only edge disorder, ballistic segments $\eta=W / \sin \alpha$ much longer than the sample width appear with the probability $\sim \eta^{-2}$ for $\eta \gg W$. Below we report the results of numerical simulations of quantum transport in quasiballistic wires with edges which are rough on an atomic scale. We analyze the effects of superdiffusion on the correlation properties of mesoscopic conductance fluctuations, $\delta G(\epsilon, B)$, and the effect of a magnetic field on the localization length, $L_{\mathrm{c}}$. To anticipate a little, the correlation energy of conductance fluctuations we found scales anomalously with the system size as $E_{\mathrm{c}}=\frac{1}{2} h W v_{F} L^{-2} \times$ $\ln \left(\frac{L}{1.7 \mathrm{~W}}\right)$. Although geometrical flux cancellation [8-10] hinders the conventional crossover in the localization effects between the orthogonal and unitary symmetry classes $[17,18]$, we observe the usual Aharonov-Bohm magnetoconductance fluctuations due to the deflection of Lévy flights by a magnetic field (which transforms part of them into skipping orbits). This deflection breaks the flux cancellation rules and also determines the magnetic field dependence of the localization length which is different from that obtained in ergodic (with bulk impurities) disordered systems.

The results presented below are based upon numerical solution of a two-dimensional Anderson Hamiltonian on a square lattice, $H=\sum_{i}|i\rangle \epsilon_{i}\left\langle i\left|-V \sum_{\langle i j\rangle}\right| i\right\rangle\langle j|$, where $\langle i j\rangle$ denotes nearest neighbor sites $i$ and $j$. The structure considered consists of two ideal leads of width $W$ attached to a scattering region $W$ sites wide and $L$ sites long (all lengths are in units of the lattice constant $a$ ). In the absence of a magnetic field, the off-diagonal matrix elements $V=1$ determine the width of the energy band. Within the leads and in the center of the scattering region (when modeling clean wires with rough boundaries), the diagonal matrix elements were $\epsilon_{i}=\epsilon_{0}$ (with $\epsilon_{0}>1$, which keeps the Fermi level away from the van Hove singularity in the band center). For simulating bulk disorder, the $\epsilon_{i}$ in the scattering region are taken uniformly from the interval $-U / 2<\epsilon_{i}-\epsilon_{0}<U / 2$ where $U$ is the disorder strength. For sites on the boundary, $\epsilon_{i}=\epsilon_{0}+\epsilon_{B}$ where $\epsilon_{B}=10^{4}$. The rough structure of the boundary was 
generated by having an equal probability of either 0,1 , or 2 sites at each edge with on-site potential $\epsilon_{0}+\epsilon_{B}$ [19]. To simulate the effect of a magnetic field, we incorporate a Peierls phase factor into $V$ in the scattering region. Numerical decimation techniques [20] were used to compute the Green's function for each given realization of disorder. From these the transmission matrix $t t^{\dagger}$ was found and diagonalized. Its eigenvalues, $T_{n}$, were analyzed statistically and used to compute the conductance $G=\sum_{n} T_{n}$, which we measure in units of $\frac{e^{2}}{h}$. Note that numerical results presented here describe a model system without any decoherence (i.e., without any inelasticity).

The effect of Lévy flights can be easily identified in our numerical results of quantum transport in pure wires with edge disorder. Rather than consider the statistics of the $T_{n}$ 's it is more useful to introduce the parametrization $T_{n}=1 / \cosh ^{2}\left(L / \xi_{n}\right)$ and consider the distribution, $P\left(\xi^{-1}\right)$, of inverse localization lengths or Lyapunov exponents. This is shown in Fig. 1(a) for four series of quasiballistic wire samples with $W=15$ (giving a mean width of 13) and lengths $L=52(A), 104(B), 208(C)$, and $416(D)$, and a series of samples $(U)$ with bulk "defects." As pointed out by Tesanovich et al. [7] and verified numerically in Ref. [11], the length of Lévy flights, $\eta_{\max }$, is limited in quantum systems. This is because uncertainty in the transverse momentum, $\delta k_{\perp} \sim \hbar / W$, in a wire with a finite width sets a quantum limit to the angles $W / \eta \sim \alpha>\delta \alpha \sim \delta k_{\perp} / k_{F} \sim \lambda / W$ which can be assigned to a classically defined ballistic segment [10]. This sets the cutoff $\eta_{\max }=W^{2} / \lambda_{F}$, and a finite localization length $L_{\mathrm{c}} \sim \frac{\pi W^{2}}{\lambda_{F}}$. Samples from the series $A$ and $B$ meet the criterion $L<L_{\mathrm{c}}$, and the distribution of the Lyapunov exponents obtained in them has a pronounced peak at small $\xi^{-1}$ corresponding to eigenvalues $T_{n} \approx 1$. This should be compared to the plateauxlike [21] distribution $P\left(\xi^{-1}\right)$ reproduced using the same numerical procedure in a sample from the series $U$. Note a finite width of the ballistic peak in $P\left(\xi^{-1}\right)$ and that $P(0)=0$. The enhanced density of small $\xi^{-1}$ can be identified even in samples from the series $C$ and $D$ with $L \approx L_{\mathrm{c}}$, where $P\left(\xi^{-1}\right)$ starts to show a periodic modulation specific to the localized regime where the spectrum of Lyapunov exponents tends to crystallize $[18,22]$.

The distribution of the eigenvalues of the transmission matrix results in a finite-width distribution of conductances and, hence, conductance fluctuations. The statistics of conductance fluctuations, $P(G)$, for wires with edge disorder is shown in Fig. 1(b) (for the case of zero magnetic field) for the series $A, W=15, L=52<L_{\mathrm{c}} \sim$ $\pi W^{2} / \lambda_{F} \sim 200,\langle G\rangle \approx 2.0$ (the distribution is almost Gaussian), and $C, W=15, L=208 \sim L_{\mathrm{c}},\langle G\rangle \approx 0.71$. The distribution function $P(G)$ is the result of the analysis of various realizations of disorder. We shall denote such averaging over realizations by $\langle\cdots\rangle$. When calculating correlation functions, we also add averaging over energy. The sample length dependence of the correlation energy, $E_{\mathrm{c}}(L)$, of the fluctuation pattern found from the half-
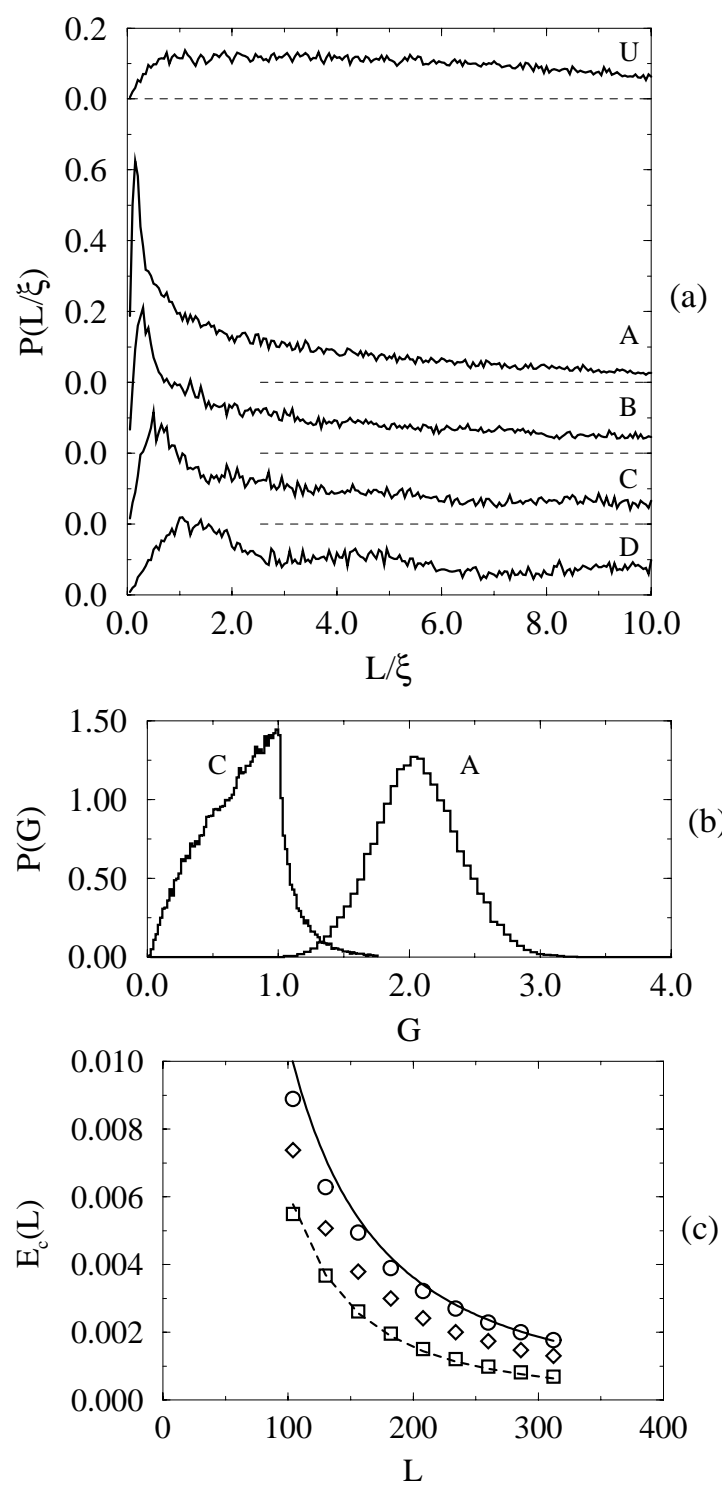

FIG. 1. (a) Distribution function $P(L / \xi)$ for four quasiballistic samples with $W=15$ and $L=52(A), 104(B), 208(C)$, and $416(D)$ and for one wire $(U)$ with on-site bulk disor$\operatorname{der} U=2.0$ (nominal mean free path $l \approx 8.5, W=15$, and $L=52$ ). (b) Shows $P(G)$ for the two quasiballistic structures $A$ and $C$. (c) Shows $E_{\mathrm{c}}(L)$ for quasiballistic wires with $\epsilon_{0} \in[1.5,1.7]$ (circles) and $\epsilon_{0} \in[1.0,1.2]$ (diamonds) and for a wire from the $U$ series (squares). Also shown is the analytical result [Eq. (2), solid curve] with $h v_{f}=12.3$ and $W=9.3$ [15]. The dashed curve is the ergodic law $E_{\mathrm{c}} \sim h D / L^{2}$ with $h D=62.5$.

height of the correlation function $\langle\delta G(\epsilon) \delta G(\epsilon+\delta \epsilon)\rangle$ is shown in Fig. 1(c). For comparison, $E_{\mathrm{c}}(L) \sim L^{-2}$ is also shown for bulk-disordered samples $(U)$, which serves us as a reference case.

In wires with edge disorder ballistic segments $\eta=$ $W / \sin \alpha$ much longer than the sample width appear with the probability $\pi^{-1}\left|\frac{d \eta(\alpha)}{d \alpha}\right|^{-1} \sim \eta^{-2}$ for $L>\eta \gg W \gg$ $\lambda_{F}$, and this gives rise to a difference of the correlation energy sample length dependence from the aforementioned ergodic law. The correlation energy is determined by 
the lowest eigenvalue in the relaxation spectrum of the diffusion operator. In the anomalous case, the diffusion process is described by the integral equation

$$
\begin{aligned}
P\left(\frac{t}{\tau} ; x, x^{\prime}\right)= & \frac{1}{2} \int_{-L / 2 W}^{L / 2 W} \\
& \times \frac{P\left(\frac{t}{\tau}-\sqrt{1+(x-y)^{2}} ; y, x^{\prime}\right)}{\left[1+(x-y)^{2}\right]^{3 / 2}} d y
\end{aligned}
$$

with initial conditions $P\left(t=0 ; x, x^{\prime}\right)=\delta\left(x-x^{\prime}\right)$. The correlation function $P\left(t / \tau ; x, x^{\prime}\right)$ describes the probability density to find a particle at the diffusively scattering edge of a sample symmetrized over both wire edges, and it is represented in the coordinates along the wire axis normalized by the wire width. The time is normalized by the ballistic time, $\tau=W / v_{F}$. The solution of this equation in a long system can be found by taking the Fourier transform,

$P(\omega, q)=\left[1-\int_{-\infty}^{\infty} \frac{\exp \left(i q x+i \omega \sqrt{1+x^{2}}\right)}{2\left[1+x^{2}\right]^{3 / 2}} d x\right]^{-1}$.

To study the low-lying spectrum of $P(\omega, q)$, we expand $P^{-1}$ over $\omega \ll 1$, and find that in the limit of $q \ll 1$

$$
P^{-1} \approx-i \frac{\pi}{2} \omega+\frac{1}{2} q^{2} \ln \left(\frac{2 e^{1 / 2-\gamma}}{q}\right) .
$$

For a sample of length $L$, we take $q_{\min }=\pi W / L$ and get

$$
E_{\mathrm{c}} \sim \frac{h v_{F} W \ln (L / c W)}{2 L^{2}}, \quad c=\frac{\pi}{2} e^{\gamma-1 / 2} \approx 1.7,
$$

where $\gamma$ is Euler's constant. The anomalous dependence in this analytical result describes a faster escape of a particle from a quasiballistic wire, as compared to a bulkdiffusive one, and it is represented by the solid curve in Fig. 1(c). Note that to relate the above semiclassical analysis to purely quantum numerical simulations, we use an effective sample width $\langle W\rangle-\lambda_{F}$. In addition, it is possible to calculate the variance of conductance fluctuations in a quasiballistic case, $\left\langle\delta G_{\mathrm{qb}}^{2}\right\rangle$. This has been done by evaluating the probabilities of two paths to encounter each other at two scatterers, which uses the result of Eq. (1). Such a calculation shows that $\left\langle\delta G_{\mathrm{qb}}^{2}\right\rangle /\left\langle\delta G_{\mathrm{erg}}^{2}\right\rangle=\frac{64}{\pi^{4}} \approx 0.66$, whereas the numerical result obtained for a system with $W=15$ and $L=104$ is $\left\langle\delta G_{\mathrm{qb}}^{2}\right\rangle /\left\langle\delta G_{\mathrm{erg}}^{2}\right\rangle \approx 0.72$.

Another feature of quasiballistic wires is flux cancellation [8-10], which is an exact geometrical fact in ballistic systems with parallel edges. Because of this cancellation, no Aharonov-Bohm flux can be encircled by loops composed of a sequence of ballistic flights between sample boundaries. In the metallic regime, $L<L_{\mathrm{c}}$, the curving of an electronic trajectory by a magnetic field reduces the length of the longest ballistic paths, so that the conductance $\langle G\rangle$ declines when the transverse deflection of the Lévy flights, $\delta x_{\perp} \approx \frac{1}{2}\left(e v_{F} B / m c\right)\left(L / v_{F}\right)^{2} \sim B L^{2} \lambda_{F} / \phi_{0}$ becomes larger than the wire width, $\delta x_{\perp} \geq W$, at

$$
B>B_{\text {def } 1} \sim \phi_{0} W /\left(\lambda_{F} L^{2}\right) \sim\left(L_{\mathrm{c}} / L\right)(W L)^{-1} \phi_{0} .
$$

This effect is present both in the averaged (over disorder) conductance for samples from series $A$ and $B$ shown in Fig. 2(a) and in the suppression of a peak at small $\xi^{-1}$ in $P\left(\xi^{-1}\right)$ in Fig. 2(b) for samples from the same series, as in Fig. 1(a). The conductance $\langle G\rangle$ decreases up to the field where the electron cyclotron radius $R_{\mathrm{c}}$ becomes commensurable with the wire width $W$. At such a field, $B_{\text {com }}=$ $0.55 \frac{\hbar k_{f}}{e W}$ [14], the magnetoconductance has a minimum followed by a rise and formation of the quantum Hall effect plateaux after which any electron cyclotron orbit either decouples from the edges or skips independently along each of them [23].

The shortening of the length of Lévy flights reduces the localization length in a wire. In samples from the series $C$ and $D$ [also shown in Fig. 2(b)], localization appears to be stronger in a field than at $B=0$ : The crystallization in the spectrum of $\xi^{-1}$ prevails, which reflects the fact, supported by the analysis of longer wires (up to $L=$ 1040), that the localization length is shortened by a field. This tendency, illustrated in Fig. 2(c) (solid curve), is opposite to the behavior of $L_{\mathrm{c}}(B)[17,18]$ in diffusive
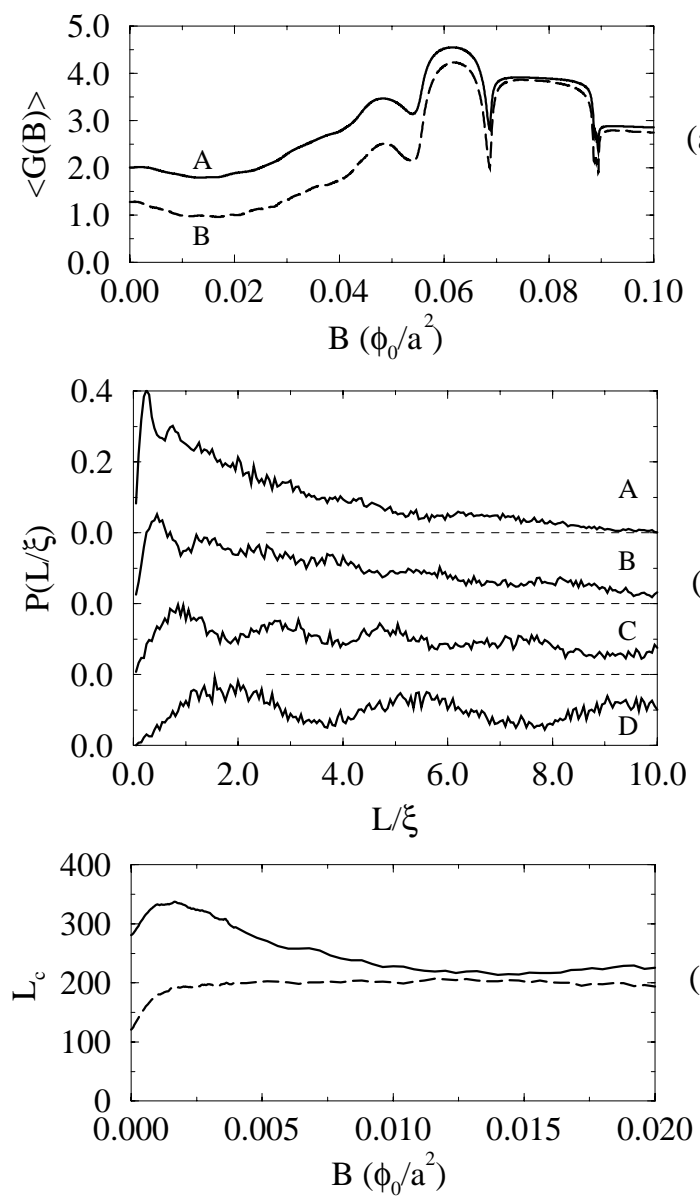

(c)

FIG. 2. (a) The ensemble averaged conductance as a function of a magnetic field for wires from the $A$ and $B$ series. (b) The same curves $(A)-(D)$ as for Fig. 1(b) but at finite magnetic field $B=0.02 \phi_{0} / a^{2}$. (c) The localization length for quasiballistic (solid curve) and disordered (dashed curve, bulk mean free path $l \approx 8.5$ ) wires. 


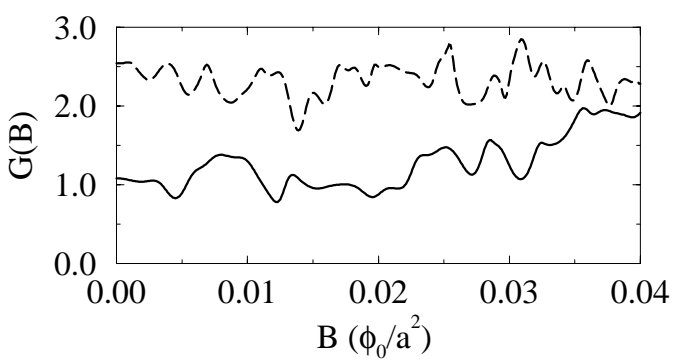

(a)

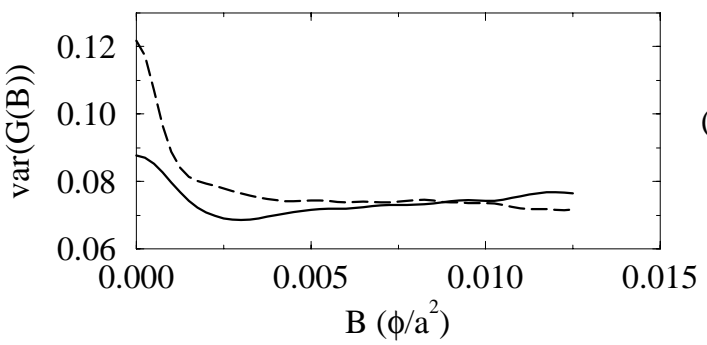

FIG. 3. (a) Conductance for one quasiballistic wire (solid curve) from the $B$ series and one disordered wire (dashed curve, mean free path $l \approx 16$ ). (b) Variance $\operatorname{var}[G(B)]$ for quasiballistic wires from the $B$ series (solid curve) and disordered wires (dashed curve, mean free path $l \approx 16$ ).

samples from the $U$ series (dashed curve). The relevant scale of magnetic fields at which the tendency of the localization length to shorten in quasiballistic wires starts, $\phi_{0} \lambda_{F} / W^{3}$, can be obtained from $B_{\text {defl }}$ by replacing the sample length $L$ with $L_{\mathrm{c}}$, which formally gives a similar scale as a field which would provide the Aharonov-Bohm type of a crossover in $L_{\mathrm{c}}$ between two symmetry classes, so that the latter is hindered by the deflection effect.

Deflection of Lévy flights at $B>B_{\text {defl }}$ also breaks the geometrical flux cancellation and restores the ability of a pair of electron paths to encircle a magnetic flux $\sim L W B$. At this field the longest ballistic flights, which still dominate transport, transform into skipping orbits so that the electron path inside a sample acquires segments which do not cross the wire: They start and end on the same edge. On the one hand, the positive magnetoconductance which could result from the suppression of the weak localization correction at the field scale of $B \sim B_{\text {defl }}$ is mostly hindered by a larger negative classical effect. On the other hand, the breakdown of geometrical flux cancellation manifests itself in pronounced magnetoconductance fluctuations $\delta G(B)=G(B)-\langle G\rangle$ illustrated in Fig. 3(a) (solid line), which shows the conductance $G(B)$ of a single sample, along with results from a sample from the $U$ series (dashed line-this has been shifted by 1 vertically for clarity). The variance of fluctuations is shown in Fig. 3(b). From analyzing the autocorrelation function we deduce the sample length dependence of the correlation field $B_{\mathrm{c}}$ in quasiballistic samples. It corresponds to the magnetic field flux through the sample area equal to $2.5 \phi_{0}$, in comparison with $1.5 \phi_{0}$ that we get in the bulk-disordered case. This seems to explain an earlier experimental observation [24].
The authors thank C.W. J. Beenakker for discussions. V.F. acknowledges financial support from EPSRC.

[1] Mesoscopic Effects in Solids, edited by B.L. Altshuler, P. A. Lee, and R. Webb (North-Holland Publ., Amsterdam, 1992).

[2] M. V. Berry, Proc. R. Soc. London A 400, 229 (1985).

[3] M. Gutzwiller, Chaos in Classical and Quantum Mechanics (Cambridge University Press, Cambridge, 1992).

[4] A. V. Andreev et al., Phys. Rev. Lett. 76, 3947 (1996).

[5] I. L. Aleiner and A. I. Larkin, Phys. Rev. B 54, 14423 (1996).

[6] A. McGurn and A. Maradudin, Phys. Rev. B 30, 3136 (1984).

[7] Z. Tesanovich et al., Phys. Rev. Lett. 57, 2760 (1986).

[8] V. K. Dugaev and D. E. Khmelnitskii, Sov. Phys. JETP 59, 1038 (1984).

[9] C. W. J. Beenakker and H. van Houten, Phys. Rev. B 37, 6544 (1988); 38, 3232 (1988).

[10] V. I. Falko, J. Phys. Condens. Matter 2, 3797 (1990).

[11] K. Nikolic and A. MacKinnon, Phys. Rev. B 50, 11008 (1994).

[12] N. Zhu, Hong Guo, and R. Harris, Phys. Rev. Lett. 77, 1825 (1996).

[13] J. Sanchez-Gil et al., Phys. Rev. Lett. 80, 948 (1998).

[14] A. B. Pippard, Magnetoresistance in Metals (Cambridge University Press, Cambridge, 1989).

[15] J. P. Bouchaud and A. Georges, Phys. Rep. 195, 127 (1990).

[16] Lévy Flights and Related Topics in Physics, edited by M.F. Shlesinger, G. M. Zaslavsky, and U. Frisch (Springer-Verlag, Berlin, 1994).

[17] K. B. Efetov, Supersymmetry in Disorder and Chaos (Cambridge University Press, Cambridge, 1997).

[18] J.-L. Pichard et al., J. Phys. (Paris) 51, 587 (1990); A. D. Stone et al., in Ref. [1].

[19] With this choice the mean width of the scattering region along its length is $W-2$. Note that the dispersion relation is $E=\epsilon_{0}-2 V\left[\cos \left(k_{x} a\right)+\cos \left(k_{y} a\right)\right]$ and for Lévy flights we take the Fermi velocity to be that along the wire axis (i.e., $\left.k_{y}=0\right) \hbar v_{F}=2 V a \sin \left(k_{x} a\right)$. The choice of $E=0, \epsilon_{0}=1.6 \mathrm{~V}$ leads to $\lambda_{F} \approx 3.7 a$ and $\hbar v_{F}=1.96 \mathrm{Va}$, and for $E=0, \epsilon_{0}=1 \mathrm{~V}$ we have $\lambda_{F} \approx$ $3.2 a$ and $\hbar v_{F}=1.74 V a$.

[20] C. J. Lambert and D. Weaire, Phys. Status Solidi B 101, 591 (1980).

[21] J. T. Chalker and A. Macedo, Phys. Rev. Lett. 71, 3689 (1993); C. W. J. Beenakker and B. Rejaei, ibid. 71, 3689 (1993).

[22] K. Frahm, Phys. Rev. Lett. 74, 4706 (1995).

[23] T. Ando and H. Tamura, Phys. Rev. B 46, 2332 (1992); H. Akera and T. Ando, ibid. 43, 11676 (1991). The latter happens at $B_{m} \approx 2 \frac{\phi_{0}}{\lambda_{F} W}$ which is $0.05 \phi_{0} / a^{2}$ for $\epsilon_{0} \approx 1$, and we estimate $B_{\text {com }} \approx 0.0135 \phi_{0} / a^{2}$, in agreement with numerically found values.

[24] H. van Houten et al., Superlattices Microstruct. 3, 497 (1988). 